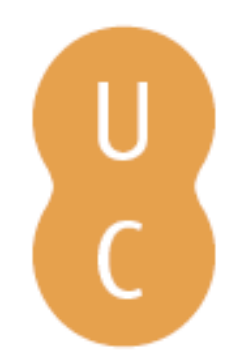

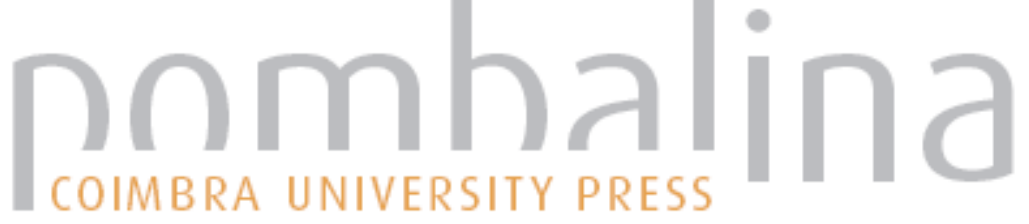

\section{Irreversibilidade, probabilidade e leis da natureza}

Autor(es): $\quad$ Petrosky, Tomio

Publicado por: Imprensa da Universidade de Coimbra; Gradiva

URL

persistente:

URI:http://hdl.handle.net/10316.2/32687

DOI:

DOI:http://dx.doi.org/10.14195/978-989-26-0389-6_14

Accessed : $\quad$ 26-Apr-2023 15:43:19

A navegação consulta e descarregamento dos títulos inseridos nas Bibliotecas Digitais UC Digitalis, UC Pombalina e UC Impactum, pressupõem a aceitação plena e sem reservas dos Termos e Condições de Uso destas Bibliotecas Digitais, disponíveis em https://digitalis.uc.pt/pt-pt/termos.

Conforme exposto nos referidos Termos e Condições de Uso, o descarregamento de títulos de acesso restrito requer uma licença válida de autorização devendo o utilizador aceder ao(s) documento(s) a partir de um endereço de IP da instituição detentora da supramencionada licença.

Ao utilizador é apenas permitido o descarregamento para uso pessoal, pelo que o emprego do(s) título(s) descarregado(s) para outro fim, designadamente comercial, carece de autorização do respetivo autor ou editor da obra.

Na medida em que todas as obras da UC Digitalis se encontram protegidas pelo Código do Direito de Autor e Direitos Conexos e demais legislação aplicável, toda a cópia, parcial ou total, deste documento, nos casos em que é legalmente admitida, deverá conter ou fazer-se acompanhar por este aviso.

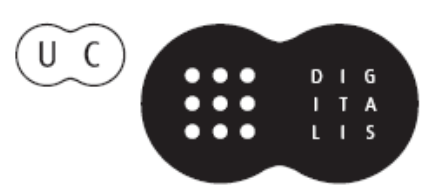


C I E N C I A I B E R T A

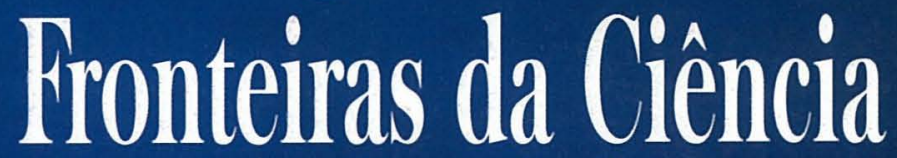

Desenvolvimentos Recentes - Desafios Futuros

RUI FAUSTO • CARLOS FIOLHAIS • JOÃO FILPE QUURRÓ

Coordenadores

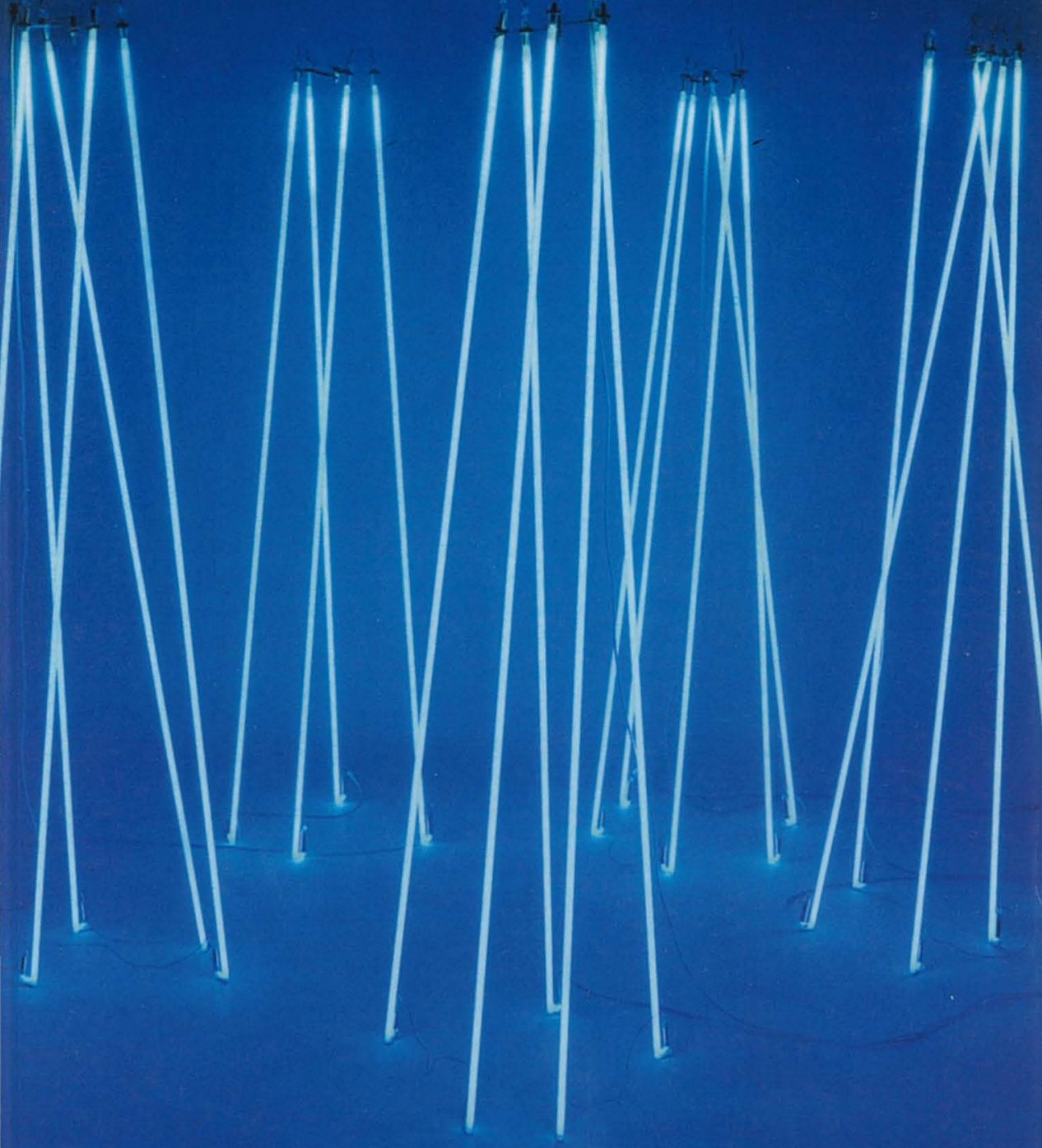

gradiva - Imprensa da Universidade de Coimbra 
(Página deixada propositadamente em branco) 


\section{RUI FAUSTO, CARLOS FIOLHAIS JOÃO FILIPE QUEIRÓ \\ Coordenadores}
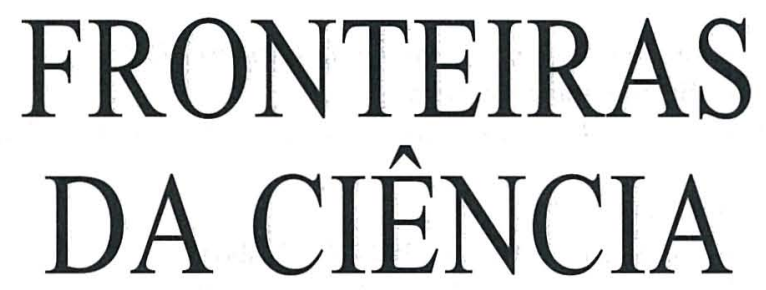

Desenvolvimentos Recentes Desafios Futuros 
(C) Gradiva - Publicações, L. da / Imprensa da Universidade de Coimbra, 2003 Coordenação editorial: Rui Fausto, Carlos Fiolhais e João Filipe Queiró Tradução: Jean Burrows, Vivien Burrows, Rui Fausto, Carlos Fiolhais e João Filipe Queiró

Revisão do texto: Isabel Pedrome

Capa: António Barros [Imprensa da Universidade. Coimbra], sobre imagem de «Águas Vivas», escultura de Silvestre Pestana, 2001

Foto: António Alves; Infografia: ESTÍMULUS [design]; Cortesia: Galeria Alvarez-Arte Contemporânea

Paginação: António Resende e Paula Isabel Jorge

Impressão e acabamento: G.C. - Gráfica de Coimbra, L. ${ }^{d a}$

Reservados os direitos para Portugal por:

Gradiva - Publicações, L. ${ }^{\text {da }}$ e Imprensa da Universidade de Coimbra

Gradiva - Publicações, L.da

Rua Almeida e Sousa, 21, r/c, esq.•1399-041 Lisboa

Telefs. $213974067 / 8 \cdot 213971357 \cdot 213953470$

Fax $213953471 \cdot$ Email: gradiva@ip.pt

URL: http://www.gradiva.pt

Imprensa da Universidade de Coimbra

Rua Antero de Quental, 195 • 3000-033 Coimbra

Telefs. 351239853110

Fax 3512398531 19・e-mail: fjrpress@ci.uc.pt

URL: http://www.imp.uc.pt

ISBN: 972-662-923-3

1." edição: Agosto de 2003

Depósito legal n. ${ }^{\circ} 199$ 463/2003 


\section{Tomio Petrosky}

Center for Studies in Statistical Mechanics and Complex Systems

Universidade do Texas, Austin, Texas, E.U.A.

Ilya Prigogine

International Solvay Institutes for Physics and Chemistry

Bruxelas, Bélgica

\section{Irreversibilidade, probabilidade e leis da natureza}

Nos anos mais recentes tem-se assistido na ciência a uma mudança radical de perspectiva a partir da percepção de que grandes classes de sistemas podem exibir transições abruptas, uma multiplicidade de estados, estruturas coerentes ou um movimento aparentemente errático caracterizado pela imprevisibilidade, muitas vezes designado por caos determinista. A ciência clássica enfatizou a estabilidade e o equilíbrio; agora vemos instabilidades, flutuações e tendências evolucionárias numa variedade de áreas que vão da física atómica e molecular, passando pela mecânica de fluidos, química e biologia, aos sistemas de grande escala que são relevantes nas ciências ambientais e económicas ${ }^{1}$. Conceitos como estruturas dissipativas e auto-organização tornaram-se muito populares. A distância ao equilíbrio e, portanto, a seta do tempo desempenham um papel essencial nestes processos, um pouco como a temperatura na física do equilíbrio. Quando baixamos a temperatura obtemos sucessivamente novos estados da matéria. Na física e na química do não-equilíbrio, quando mudamos a distância ao equilíbrio, o comportamento observado é ainda mais variado.

Como podem estas descobertas ser interpretadas do ponto de vista das leis básicas da física? A dinâmica newtoniana, tal como a relatividade e a física quântica, não fazem qualquer distinção entre o passado e o futuro. 
O tempo é simplesmente um parâmetro contabilístico sem nenhum sentido preferencial.

Desde Boltzmann que esta observação tem conduzido a infindáveis controvérsias. Não vamos tentar sumariar os vários argumentos que têm sido usados; mencionemos apenas o argumento cosmológico de Boltzmann, que foi repetido por muitos autores. Por exemplo, nas suas Lectures on Physics, Feynman escreveu: Por qualquer razão, o universo num dado momento tinha uma entropia muito baixa... e essa é a origem de toda a irreversibilidade... Sempre achámos este argumento um pouco estranho. Qualquer que seja a história passada do nosso universo, hoje observamos tanto processos simétricos relativamente à inversão do tempo como processos irreversíveis. O nosso problema consiste em compreender a origem desta diferença, que existe no momento actual, quaisquer que sejam as hipóteses em cosmologia.

Sabemos, desde Gibbs e Einstein, que, para relacionar dinâmica e termodinâmica, temos de introduzir a noção de ensembles, caracterizados por uma distribuição de probabilidade $\rho$ (a matriz densidade da mecânica quântica). Como é bem conhecido, $\rho$ satisfaz a equação de Liouville-von Neumann

$$
i \frac{\partial \rho}{\partial t}=L \rho
$$

cuja solução é

$$
U_{t} \rho(\omega)=\rho\left(S_{-t}(\omega)\right)
$$

Para Gibbs e Einstein, a introdução de ensembles era necessária devido à nossa ignorância das condições iniciais exactas. Expressava a nossa ignorância. Se é certo que a descrição estatística foi um passo decisivo, ela não resolveu o problema da irreversibilidade (ver secção seguinte). Por esta razão, algumas características adicionais são necessárias. Além do $\rho$ de grão fino, os Ehrenfests introduziram uma distribuição de grão grosso, $\rho_{\text {cg }}$ que supostamente conduzia à irreversibilidade. Este engrossamento de grão (coarse graining) introduz, contudo, perda de informação e não pressupostos dinâmicos. Tornaria a irreversibilidade o resultado das nossas aproximações. No entanto, a dinâmica tem conhecido um progresso considerável. Desde o tempo de Boltzmann, dispomos da distinção de Poincaré entre sistemas integráveis e não integráveis, temos a teoria KAM e estamos familiarizados com o caos determinista.

Ao mesmo tempo, sob o impulso da teoria quântica, tem-se realizado um grande progresso na teoria espectral. Tradicionalmente o espaço 
funcional de interesse era o espaço de Hilbert de funções de quadrado integrável. No entanto, desde os trabalhos de L. Schwartz, I. Gelfand e outros $^{2}$, podemos estudar espaços mais gerais que envolvem distribuições. É curioso que, numa carta a G. Birkhoff (de 13 de Novembro de 1935), J. von Neumann tenha escrito: Gostaria de fazer uma confissão que pode parecer imoral: eu já não acredito no espaço de Hilbert ${ }^{3}$. Como mencionaremos adiante, P. Dirac chegou à mesma conclusão.

A nossa principal razão para reconsiderar o problema da irreversibilidade a partir de um ponto de vista mais fundamental proveio do nosso trabalho na física do não equilíbrio. É bem conhecido que longe do equilíbrio podem ocorrer novas estruturas no espaço-tempo (as estruturas dissipativas ${ }^{4}$ ). A distância ao equilíbrio e, portanto, a seta do tempo desempenham um papel essencial construtivo. Mais ainda, as bifurcações longe do equilíbrio envolvem processos probabilísticos, em contradição com o carácter determinístico das leis tradicionais da física. Também a irreversibilidade aparece não apenas relacionada com a termodinâmica, mas também em muitos outros níveis de observações, como na radioactividade, na emissão espontânea, para já não falar na entropia dos buracos negros. Assim, é difícil negar à irreversibilidade um carácter dinâmico fundamental. O nosso programa foi, portanto, reformular as leis microscópicas da física de modo a incluir a probabilidade e a quebra da simetria no tempo para algumas classes bem definidas de sistemas. No livro ${ }^{5}$, um de nós escreveu: Resumidamente, a formulação usual da mecânica clássica (ou quântica) ficou «embebida» numa estrutura teórica maior, que também permite a descrição de fenómenos irreversiveis.

Acreditamos que este programa está agora, em grande medida, realizado. Queremos dar aqui uma perspectiva breve de alguns aspectos importantes. Claro que é impossível incluir neste artigo qualquer demonstração. Para isso, o leitor tem de consultar as referências originais. Além disso, este artigo é escrito do ponto de vista de um físico. Alguns aspectos matemáticos mereceriam certamente uma consideração mais profunda. Estamos ainda no início da análise funcional de espaços não normalizados envolvendo funções generalizadas ou distribuições.

\section{TRAJECTÓRIAS VERSUS ENSEMBLES}

Começamos por apresentar a questão: qual é a relação entre a formulação «individual» baseada em transformações pontuais $S_{\mathrm{t}}$ e a formulação estatística? O famoso artigo de B. O. Koopman «Sistemas 
hamiltonianos e transformações no espaço de Hilbert» (1931) fornece uma resposta parcial ${ }^{6}$. Às transformações pontuais corresponde um operador unitário $U_{\mathrm{t}}$ tal que

$$
U_{t} \rho(\omega)=\rho\left(S_{-t}(\omega)\right)
$$

para todas as funções $f$ nos espaços de Hilbert. O converso do lema de Koopman foi estabelecido por K. Goodrich, K. Gustafson e B. Misra cerca de 50 anos depois ${ }^{7}$ : a existência de um operador unitário que preserva a positividade no espaço de Hilbert assegura a existência de transformações pontuais $S_{\mathrm{t}}$. Um exemplo simples da equivalência entre a descrição individual e a estatística é dado pela equação de Liouville na forma

$$
e^{-i L t} \rho(\omega)=\rho\left(e^{+i L t} \omega\right)
$$

Parece, portanto, que a introdução da formulação estatística não conduz a nenhum conhecimento novo: $L$ é um operador hermítico no espaço de Hilbert das funções de quadrado integrável e, portanto, só tem valores próprios reais. $\mathrm{O}$ passado e o futuro desempenham assim um papel idêntico. Contudo, como veremos, quando formos além da formulação convencional baseada no espaço de Hilbert, a equivalência entre as duas descrições é quebrada para algumas classes bem definidas de sistemas dinâmicos. A formulação estatística conduz a novas soluções que, curiosamente, introduzem a probabilidade como o objecto dinâmico básico e quebram a simetria temporal.

Notemos brevemente que o lema de Koopman tem um paralelo na mecânica quântica. No quadro do espaço de Hilbert,

$$
U_{t} \rho(0)=e^{-i L t} \rho(0)=e^{-i H t} \rho(0) e^{i H t}
$$

A descrição baseada na evolução dos operadores de densidade pode ser concretizada na evolução baseada no operador hamiltoniano (ambos para estados puros em que $\rho=|\psi\rangle\langle\psi|$ e para misturas ${ }^{8}$ ).

\section{Caos determinista e espaÇos de Hilbert APARelhados («RIGged»)}

Consideremos, em primeiro lugar, sistemas dinâmicos clássicos de mistura ${ }^{9}$. Um exemplo simples é o chamado mapeamento do padeiro. Como é bem conhecido, toma a metade esquerda do quadrado unitário, estica-o de um factor de 2 na direcção do eixo dos $x x$, comprime-o de 
um factor de $1 / 2$ na direcção do eixo dos yy e, em seguida, corta o lado direito do quadrado unitário colocando-o por cima (figura 1).

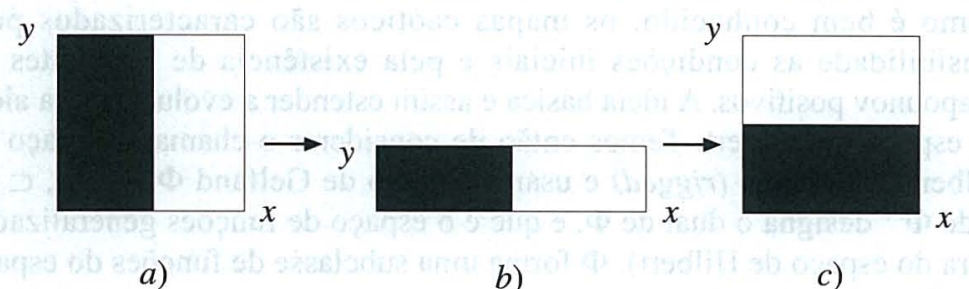

Fig. 1 - A transformação do padeiro

Para este tipo de sistema ${ }^{10}$, B. Misra, M. Courbage e I. Prigogine ${ }^{11}$ estabeleceram a conexão entre a descrição dinâmica reversível no tempo usual e a descrição probabilística por uma cadeia de Markoff, efectuando uma mudança (não unitária) de representação, sem haver qualquer perda de informação. Resumidamente, a transformação $\rho \rightarrow \tilde{\rho}=\Lambda \rho$ converte a equação de Liouville na equação

$$
i \frac{\partial \tilde{p}}{\partial t}=\Phi \tilde{p}, \quad \Phi=\Lambda L \Lambda^{-1}
$$

que representa um processo de Markoff descrevendo uma aproximação irreversível ao equilíbrio. Podemos facilmente definir uma função $H$. Não entraremos aqui na construção da transformação $L$ não unitária ${ }^{12}$. Notamos apenas que envolve o uso do operador temporal de Misra $T$, que satisfaz a relação de comutação

$$
[L, T]=-i
$$

Os nossos resultados têm sido confirmados e desenvolvidos por alguns matemáticos ${ }^{13}$. Obviamente, os sistemas de mistura são instáveis, além de que, cada região finita, seja ela de que tamanho for, contém pontos que se movem ao longo de tipos distintos de trajectórias. Escrevemos ${ }^{14}$ que tal nos força a uma nova aproximação à dinâmica. Tal está de acordo com a conclusão de von Neumann e Koopman ${ }^{15}$. O ponto importante é que a transformação $\rho \rightarrow \tilde{\rho}$ não envolve perda de informação. Não é 
necessário «engrossamento de grão» (coarse graining) ou uma outra forma de amputação da informação.

Podemos avançar nesta análise para mapeamentos que correspondem ao caos determinístico a cuja classe pertence a transformação do padeiro. Como é bem conhecido, os mapas caóticos são caracterizados pela sensibilidade às condições iniciais e pela existência de expoentes de Lyapounov positivos. A ideia básica é assim estender a evolução para além do espaço de Hilbert. Temos então de considerar o chamado espaço de Hilbert aparelhado (rigged) e usar o tripleto de Gelfand $\Phi^{+} \subset L_{2}, \subset \Phi$, onde $\Phi^{+}$designa o dual de $\Phi$, e que é o espaço de funções generalizadas (fora do espaço de Hilbert). $\Phi$ forma uma subclasse de funções do espaço de Hilbert (as funções de teste).

Esta aproximação tem sido descrita em muitos artigos, de que apresentamos aqui apenas os resultados principais ${ }^{16}$. O mapeamento do padeiro é caótico ${ }^{17}$. Existem então duas representações espectrais, sendo uma a representação usual do espaço de Hilbert e a outra envolvendo o espaço de Hilbert aparelhado, uma vez que as funções próprias são distribuições. Resumidamente, temos a representação do espaço de Hilbert que é diagonal como de costume

$$
U=\int_{0}^{2 \kappa}\left|f_{\kappa}(x, y)\right\rangle e^{i k r a}\left\langle f_{\kappa}(x, y)\right| d k
$$

e a extensão de U fora do espaço de Hilbert

$$
\mathrm{U}=\sum_{v=1}^{\infty}\left\{\sum_{\mathrm{r}=0}^{v} \frac{1}{2^{v}}\left|\mathrm{~F}_{v, \mathrm{r}}\right\rangle\left\langle\tilde{\mathrm{F}}_{v, \mathrm{r}}\left|+\sum_{\mathrm{r}=0}^{v-1}\right| \mathrm{F}_{v, \mathrm{r}+1}\right\rangle\left\langle\tilde{\mathrm{F}}_{v, \mathrm{r}}\right|\right\}
$$

onde $\mathrm{F}_{v}$ e $\tilde{\mathrm{F}}_{v}$ são distribuições. Os valores próprios $1 / 2^{v}$ estão relacionados com o tempo de Lyapounov $1 / 2$ para o mapeamento do padeiro. Observamos aqui uma nova estrutura de blocos de Jordan na decomposição espectral estendida (3.5) que não aparece no espaço de Hilbert.

Não podemos aplicar (3.5) a trajectórias individuais que são elas próprias distribuições, mas apenas a probabilidades que têm propriedades analíticas bem definidas. Mais ainda, uma vez que os espaços de funções de teste para o futuro e $o$ passado são diferentes ${ }^{18}$, a representação espectral do espaço de Hilbert aparelhado quebra a simetria temporal. Não é exagerado dizer que estes resultados são fundamentais tanto para o problema da irreversibilidade como para o papel da probabilidade na física. De facto, obtemos uma formulação das leis da física que inclui a 
quebra da simetria e que quebra também a equivalência entre trajectórias e ensembles.

Não existe, claro, nenhuma contradição entre as duas representações espectrais (3.4) e (3.5). São ambas consequências rigorosas do caos. No entanto, tal como mencionámos, diferem pelo domínio das funções onde $U$ opera.

A representação no espaço de Hilbert aparelhado contém informações adicionais importantes sobre as escalas de tempo da evolução das densidades para o equilíbrio, que se relacionam de perto com o expoente de Lyapounov. Seguimos, num certo sentido, o programa de Heisenberg da mecânica quântica. De facto, somos capazes de incluir as escalas de tempo no espectro do operador evolução.

Façamos alguns comentários para responder a certas incompreensões estranhas que apareceram na literatura. Em ambas as representações temos trajectórias. Contudo, na representação do espaço de Hilbert aparelhado as trajectórias são realizações estocásticas semelhantes ao que acontece no movimento browniano.

Em virtude da simetria temporal da equação inicial, obtemos dois semigrupos, um orientado para o futuro e o outro para o passado. Aquilo que chamamos passado e futuro não pode ser decidido considerando um mapeamento caótico isolado. O ponto principal é que os dois semigrupos estão agora separados (envolviam diferentes funções teste ${ }^{19}$ ). Em conclusão, os resultados obtidos para mapeamentos caóticos deram-nos a esperança de unificar a termodinâmica com a dinâmica. É esse assunto que vamos analisar agora.

\section{SISTEMAS TERMODINÂMICOS}

Os sistemas termodinâmicos são caracterizados por um número grande, praticamente infinito, de graus de liberdade. É apenas no limite

$$
N \rightarrow \infty, \quad V \rightarrow \infty, \quad \frac{N}{V}=C \quad \text { finito }
$$

que obtemos uma formulação correcta das transições de fase. A situação é semelhante à da teoria quântica de campos, onde também consideramos o limite de sistemas infinitos. Nas suas «Lições sobre Teoria Quântica dos Campos», P. Dirac ${ }^{20}$ escreveu: [...] se queremos considerar a totalidade dos vectores «ket» para todos os tempos, temos de ter um espaço muito maior do que o espaço de Hilbert. A interacção entre os graus de liberdade destrói a estrutura do espaço de Hilbert. A situação é 
um pouco semelhante para os sistemas termodinâmicos no limite (4.1). No limite termodinâmico (4.1), requeremos que eles sejam variáveis extensivas como a energia potencial média $U$

$$
\lim _{N \rightarrow \infty} \frac{\langle U\rangle}{N}=\text { finito }
$$

e variáveis intensivas, como a pressão $p$

$$
\lim _{N \rightarrow \infty} p=\text { finito }
$$

Em artigos recentes ${ }^{21}$, considerámos os exemplos de redes anarmónicas, assim como sistemas grandes de partículas em interacção. Sumariemos os nossos resultados para redes anarmónicas a uma dimensão. Dada a distância de equilíbrio $a$ entre dois sítios vizinhos da rede, (4.1) reduz-se neste limite a $N \rightarrow \infty$ (uma vez que $V=a N$ ).

As redes harmónicas correspondem a sistemas integráveis, uma vez que não há qualquer acoplamento entre os modos normais. A energia potencial $U$ é dada pela forma quadrática ${ }^{22}$,

$$
U-U_{0}=\frac{1}{2} \sum_{n n^{\prime}} A_{n n^{\prime}} u_{n} u_{n^{\prime}}
$$

onde $u_{\mathrm{n}}$ é o deslocamento do átomo $n$ da sua posição de equilíbrio (impomos condições fronteiras periódicas ${ }^{\mathrm{u}} \mathrm{n}+N=u_{\mathrm{n}}$ ). Podemos resolver o problema dinâmico quer por transformações pontuais usando variáveis de ângulo-acção $\alpha$ e $J$, quer pela equação de Liouville, que impõe a estrutura de espaço de Hilbert. Com notações óbvias ${ }^{23}$,

$$
\rho(J, \alpha)=\sum_{\{n\}} \rho\{n\}(J) e^{i} \sum_{\kappa} n_{\kappa} \alpha_{\kappa}
$$

A norma de Hilbert é, portanto,

$$
\|\rho\|^{2}=\int d J \sum_{\{\eta\}}\left|\rho_{\{n\}}(J)\right|^{2}
$$


Para obter uma norma de Hilbert finita para $N \rightarrow \infty$, têm de ser satisfeitas condições bem definidas. De facto, a norma (4.6) contém termos como $\left(\operatorname{com} n_{\mathrm{k}}=\ldots,-1_{\mathrm{k}}, 0,1_{\mathrm{k}}, 2_{\mathrm{k}}, \ldots\right.$, e abreviando as integrações sobre as variáveis de acção)

$$
\|\rho\|^{2}=\left|\rho_{0}\right|^{2}+\sum_{\kappa}\left|\rho_{1_{\kappa}}\right|^{2}+\sum_{\kappa \kappa^{\prime}}\left|\rho_{1_{\kappa^{\prime}} 1 \kappa^{\prime}}\right|^{2}+\sum_{\kappa \kappa^{\prime} \kappa^{\prime}}\left|\rho_{1_{\kappa^{\prime}} 1 \mathcal{K}^{\prime} 1 \mathcal{K}^{\prime \prime}}\right|^{2}+\ldots
$$

que têm de convergir para $\mathrm{N} \rightarrow \infty$. Tal implica

$$
\rho_{0} \sim 0(1), \quad \rho_{1 \kappa} \sim \frac{1}{\sqrt{N}}, \quad \rho_{1} \kappa^{1} \kappa^{\prime}, \frac{1}{N}
$$

assim como

$$
\rho_{1_{\kappa} 1_{\kappa^{\prime}} 1_{\kappa^{\prime \prime}}} \sim \frac{1}{N}
$$

para os componentes com $\mathrm{K}+\mathrm{k}^{\prime}{ }^{1}+\mathrm{k}$ " $=0$ ou um vector na rede recíproca $\mathrm{e}$

$$
\rho_{1_{\kappa} 1_{K^{\prime}} 1_{\kappa^{\prime \prime}}} \sim \frac{1}{N^{3 / 2}}
$$

para as componentes que não estão na rede recíproca. Contudo, se $\rho_{1 K^{1} K^{\prime} 1 K^{\prime \prime}}$ for muito grande, como $N^{-1 / 2}$ em vez de $N^{-1}$ (ver 4.9), a norma de Hilbert diverge.

Consideremos então redes anarmónicas. A energia potencial é, na ordem mais baixa ${ }^{24}$,

$$
U-U_{0}=\frac{1}{2} \sum_{n n^{\prime}} A_{n n^{\prime}} u_{n} u_{n^{\prime}}+\frac{1}{6} \sum_{n n^{\prime} n^{\prime \prime}} B_{n n^{\prime} n^{\prime \prime}} u_{n} u_{n^{\prime}} u_{n^{\prime \prime}}
$$

Termos de ordem mais elevada no deslocamento não acarretariam qualquer mudança. $\mathrm{O}$ hamiltoniano é então

$$
H=H_{0}+\lambda V
$$

onde introduzimos o parâmetro $\lambda$ para a constante de acoplamento. Depois de alguns cálculos ${ }^{25}$, 


$$
V=\sum_{\kappa K^{\prime} K^{\prime \prime}}\left(\frac{J_{K} J_{K^{\prime}} J_{K^{\prime \prime}}}{\omega_{\kappa} \omega_{K^{\prime}} \omega_{\kappa^{\prime \prime}}}\right)^{\frac{1}{2}}\left[V_{k k^{\prime} k^{\prime \prime}} e^{i\left(\alpha_{k}+\alpha_{k^{\prime}}+\alpha_{k^{\prime \prime}}\right)}+3 V_{k k^{\prime}-k^{\prime \prime}} e^{i\left(\alpha_{k}+\alpha_{k^{\prime}}-\alpha_{k^{\prime \prime}}\right)}+c . c .\right]
$$

com a linha (') a significar que a soma sobre $\mathrm{K}, \mathrm{K}$ ', K" se restringe a vectores na rede recíproca. Pode verificar-se facilmente ${ }^{26}$ que

$$
V_{\kappa \kappa^{\prime} \kappa^{\prime \prime}} \sim \frac{1}{\sqrt{N}}
$$

É agora fácil mostrar que a estrutura de espaço de Hilbert é incompatível com o limite termodinâmico $N \rightarrow \infty$. No equilíbrio termodinâmico, esperamos que (4.2) seja satisfeita (equipartição da energia). Em contraste, usando (4.9), correspondendo à estrutura do espaço de Hilbert, obtemos

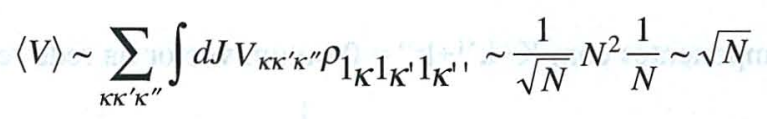

Isto mostra que o equilíbrio termodinâmico está fora do espaço de Hilbert. Para obter (4.2) necessitamos de correlações «mais fortes», tais como (4.9)

$$
\rho_{1_{\kappa} 1_{\kappa^{\prime}} 1_{\kappa^{\prime \prime}}} \sim \frac{1}{\sqrt{N}}
$$

mas então a norma do espaço de Hilbert diverge. Remetemos o leitor para os artigos originais, para a descrição do modo como $\rho$ é «ejectado» do espaço de Hilbert em resultado das interacções ${ }^{27}$.

A nossa conclusão aplica-se da mesma maneira a sistemas de partículas em interacção no limite termodinâmico. No entanto, a extensão do espaço funcional para fora do espaço de Hilbert não implica quebra de simetria temporal. Para isso necessitamos adicionalmente de não integrabilidade no sentido de Poincaré, tal como se aplica a sistemas termodinâmicos grandes (chamamos-lhes sistemas grandes de Poincaré SGP ${ }^{28}$ ). Tal como é bem conhecido, a não integrabilidade de Poincaré está associada a ressonâncias, o que conduz a novos processos na descrição estatística. 
Podemos ter processos de «destruição» (de correlações) como estão representados graficamente na figura 2 .

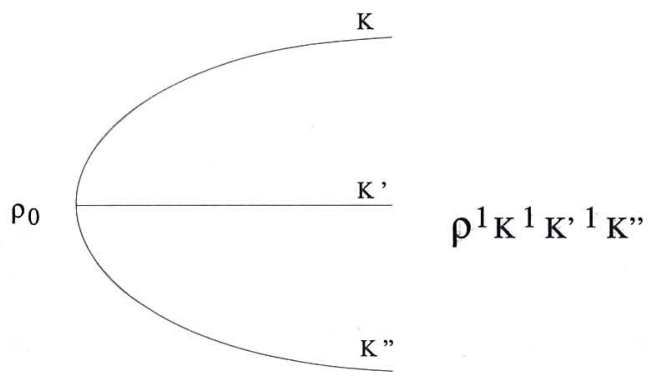

Fig. 2 - Destruição de correlações levando de correlações de três modos $\rho^{1} \mathrm{k}^{1} \mathrm{k}^{\prime 1} \mathrm{k}^{\prime \prime}$ para um vácuo de correlação

Podemos também ter processos de criação de correlações (figura 3). Em resultado das ressonâncias de Poincaré, podemos também ter processos que relacionam estados correspondendo ao mesmo grau de correlação (ou seja $\rho_{0} \operatorname{com} \rho_{0}$ ) - ver figura 4 - que chamámos processos de colisão.

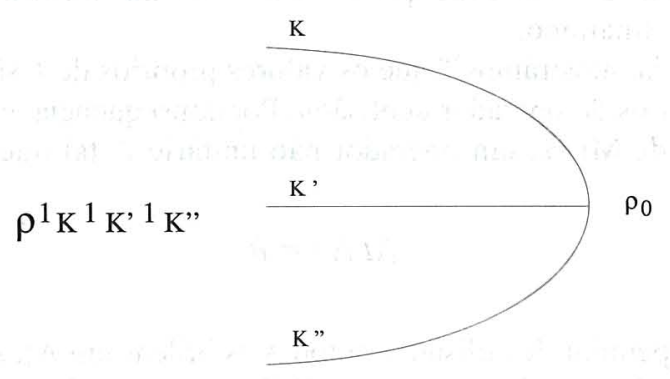

Fig. 3 - Criação de correlações levando de $\rho_{0}$ para $\rho^{1} \mathrm{k}^{1} \mathrm{k}^{\prime 1} \mathrm{k}^{\prime \prime}$ 
Os processos de colisão destroem a descrição de trajectória. Na figura 4 , cada vértice contém operadores de derivação $\partial / \partial J$. A figura 4 conduz, portanto, a processos difusivos contendo operadores de segunda ordem $\partial^{2} / \partial J^{2}$, característicos de processos difusivos.

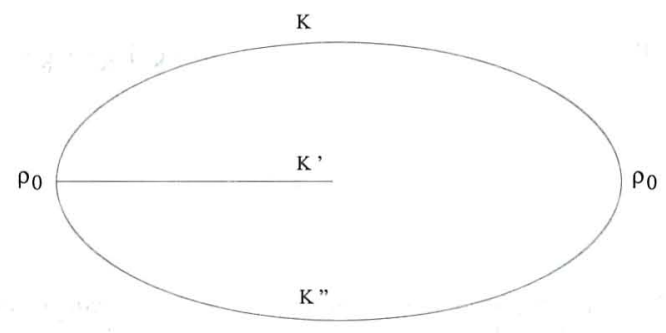

Fig. 4 - Processos de colisão relacionando $\rho_{0} \operatorname{com} \rho_{0}$

O principal resultado é que a dinâmica de SGP é governada por interacções de «tipo Langevin», tal como aparecem, por exemplo, na equação de Fokker-Planck para a dinâmica clássica e na equação de Pauli para a mecânica quântica. Podemos descrever qualitativamente a situação imaginando que cada observável corresponde a um número finito de graus de liberdade que «nadam» no mar infinito associado ao limite termodinâmico.

Mais ainda, mostrámos ${ }^{29}$ que os valores próprios de $L$ são dados pelos valores próprios do operador «colisão». Por consequência, existe, tal como no trabalho de Misra, um operador não unitário $\Lambda$ tal que

$$
\Lambda L \Lambda^{-1}=\theta
$$

onde $\theta$ é o operador de colisão (em todas as ordens em $\Lambda$ ). Assim, a nossa extensão de $L$ fora do espaço de Hilbert é semelhante ao operador dissipativo $\theta$. Exemplos assim como referências a aplicações recentes são dadas nos artigos originais ${ }^{30}$. 
Vemos que os sistemas de Poincaré grandes envolvem efeitos novos não considerados na mecânica newtoniana ou na mecânica quântica. Tal como para o caos determinista, obtemos uma formação da dinâmica baseada em probabilidades que quebram a simetria temporal. Os sistemas SGP ainda são integráveis, mas ao nível de probabilidades.

Todos estes resultados se estendem facilmente à mecânica quântica. Obtemos então uma formulação da mecânica quântica fora do espaço de Hilbert na qual as quantidades fundamentais são matrizes densidade e já não amplitudes de onda.

\section{Partículas, CAMpos e IRReVersibilidade}

A física atómica, nuclear e de altas energias trabalha com estados excitados ou partículas instáveis. Estes objectos não podem ser funções próprias do hamiltoniano (em contraste com o estado fundamental ou com partículas em estados estáveis). Dirac reconheceu bem esta situação. Escreveu ${ }^{31}$ : $O$ facto de que tivemos de usar a palavra aproximadamente ao enunciar as condições requeridas para poderem ocorrer os fenómenos de emissão e absorção mostra que estas condições não são expressáveis numa linguagem matemática exacta. Só podemos dar sentido a estes fenómenos referindo um método perturbador. Ocorrem quando o sistema não perturbado (do dispersor mais partícula) tem estados estacionários fechados. A introdução desta perturbação destrói a propriedade de estacionaridade destes estados e dá origem à emissão espontânea e à sua inversa, a absorção.

Descrevamos em pormenor esta dificuldade usando o bem conhecido modelo de Friedrichs, que descreve a interacção de um átomo de dois níveis com a radiação ${ }^{32}$.

Neste modelo temos um estado discreto|1 $\rangle$ representando uma partícula nua acoplada a estados contínuos $|\kappa\rangle$ correspondendo a modos de campo ${ }^{33}$. O hamiltoniano é

$$
H=H_{0}+\lambda V=|1\rangle \omega_{1}\left\langle 1\left|+\sum_{\kappa}\right| \kappa\right\rangle \omega_{\kappa}\left\langle\kappa\left|+\lambda \sum_{\kappa} V_{\kappa}\right| \kappa\right\rangle\langle 1|+| 1\rangle\langle\kappa|
$$

Os estados $|1\rangle$ e $|\kappa\rangle$ são funções próprias de $H_{0}$. O efeito da perturbação é levar à emissão ou absorção de um fotão (este modelo despreza transições virtuais). Existem duas situações de acordo com o sinal de $w_{1}$ (figura 5). 

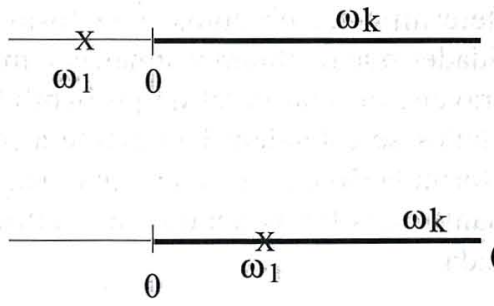

Fig. 5-a) Estado ligado

b) Ressonância

Para $\omega_{1}<0$ temos um estado ligado, para $\omega_{1}>0$ o estado $|1\rangle$ decairá emitindo um fotão devido a ressonâncias entre a partícula e o fotão. $O$ caso $\omega_{1}<0$ pode ser facilmente tratado. A interacção transforma $|1\rangle \mathrm{em}$ $\left|\phi_{1}\right\rangle$, que é um estado próprio de $H$ (para $\left.\tilde{\omega}_{1}<0\right)$ :

$$
H\left|\phi_{1}\right\rangle=\tilde{\omega}\left|\phi_{1}\right\rangle
$$

Pode demonstrar-se que a expressão exacta de $\left|\phi_{1}\right\rangle$ é $^{34}$

$$
\left|\phi_{1}\right\rangle=N_{1}^{\frac{1}{2}}\left[|1\rangle-\sum_{\kappa} \frac{\lambda V_{\kappa}}{\omega_{\kappa}-\tilde{\omega}_{1}}|\kappa\rangle\right]
$$

onde $N_{1}$ é uma constante de normalização.

A fórmula (6.3) mostra claramente a nuvem do fotão $|\kappa\rangle$, que rodeia $|1\rangle$. De modo semelhante, temos um fotão vestido. $O$ estado $|1\rangle$ evolui com o tempo para $\left|\phi_{1}\right\rangle$. A figura 6 dá um exemplo da distribuição de fotões em volta da partícula estável (na simulação numérica efectuada pelo Dr. G. Ordonez usámos $\lambda=0,1, V_{\mathrm{k}}=L^{-1 / 2}, L=400$, onde $L$ é o tamanho do sistema). Vê-se claramente a nuvem que rodeia $|1\rangle$.

Os dois picos mais pequenos que viajam para fora da partícula são sobreposições de fotões vestidos; estes fotões são criados, uma vez que 
a energia da partícula nua é maior do que a energia da partícula vestida $\omega_{1}>\tilde{\omega}_{1}$. À medida que emerge a partícula vestida, os fotões transportam para fora o excesso de energia.

Consideremos agora o caso da ressonância (ver figura 7). Friderichs mostrou que temos então a decomposição espectral

$$
H=\sum \omega_{\kappa}\left|\phi_{\mathrm{k}}^{\mathrm{F}}\right\rangle\left\langle\phi_{\mathrm{k}}^{\mathrm{F}}\right|
$$

onde os $\left|\phi_{k}^{F}\right\rangle$ são ondas a caminhar para o exterior que satisfazem as condições de completude e ortonormalidade. $\mathrm{O}$ estado excitado não aparece explicitamente em (6.4).

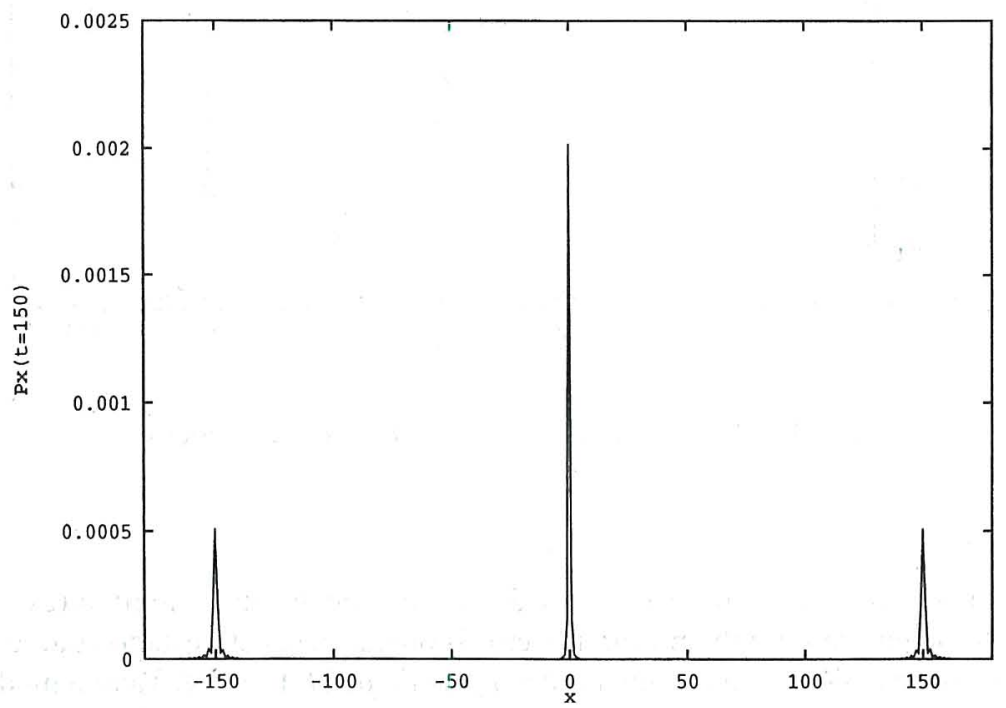

Fig. 6-Distribuição de fotões em volta de uma partícula estável em $t=150$

Como mencionámos (ver figura 5), a transição de estados ligados para ressonâncias tem lugar em $\omega_{1}=0$. Podemos visualizar a transição por meio dos gráficos computacionais da figura 7 . 
Na figura 7 representamos a distribuição espacial de fotões para três casos: a) $\left.\omega_{1}=-1, b\right) \omega_{1}=-0.01$ e c) $\omega_{1}=+0.01$. O caso $a$ ) já foi considerado por nós. Colocamo-lo aqui apenas para referência. Os casos $b$ ) e $c$ ) estão perto do ponto de ramificação $\omega_{1}=0$. O caso $b$ ) corresponde à partícula estável e $c$ ) à partícula instável.

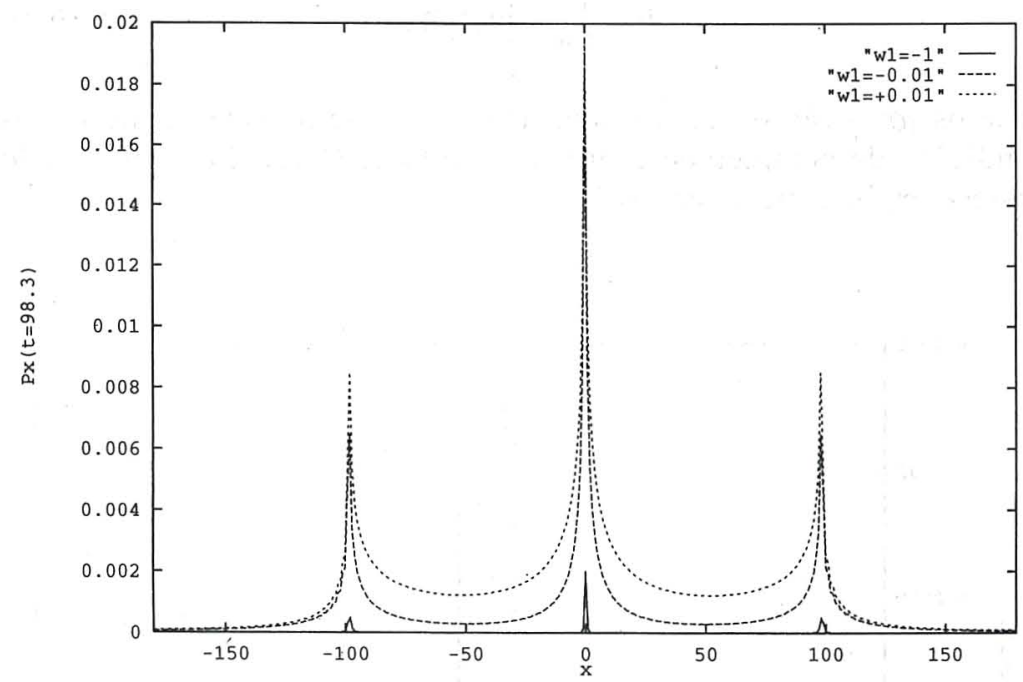

Fig. 7 - Distribuição de fotões perto do ponto de transição

É óbvio que as distribuições de fotões são muito semelhantes. A ressonância tem também uma nuvem fotónica, mas este estado (que é a continuação de $\left|\phi_{1}\right\rangle$ ) não é uma função própria de $H$. É interessante o modo como se forma este estado. Em $t=0$, temos um pacote de onda com uma forma rectangular aproximando-se do átomo no seu estado fundamental. Depois do contacto, o átomo está excitado e decai exponencialmente.

Note-se a assimetria entre o período de formação e o decaimento. Isto indica claramente o carácter irreversível do processo. A questão é a natureza do estado em decaimento (que aparece para $t=500$ na figura 8 ). Podemos aplicar a transformação não unitária (5.6) para descrever o estado em decaimento. O principal é que as ressonâncias são descritas 
por operadores densidade (e não funções de onda) que evoluem irreversivelmente. A quantidade central é novamente a probabilidade.

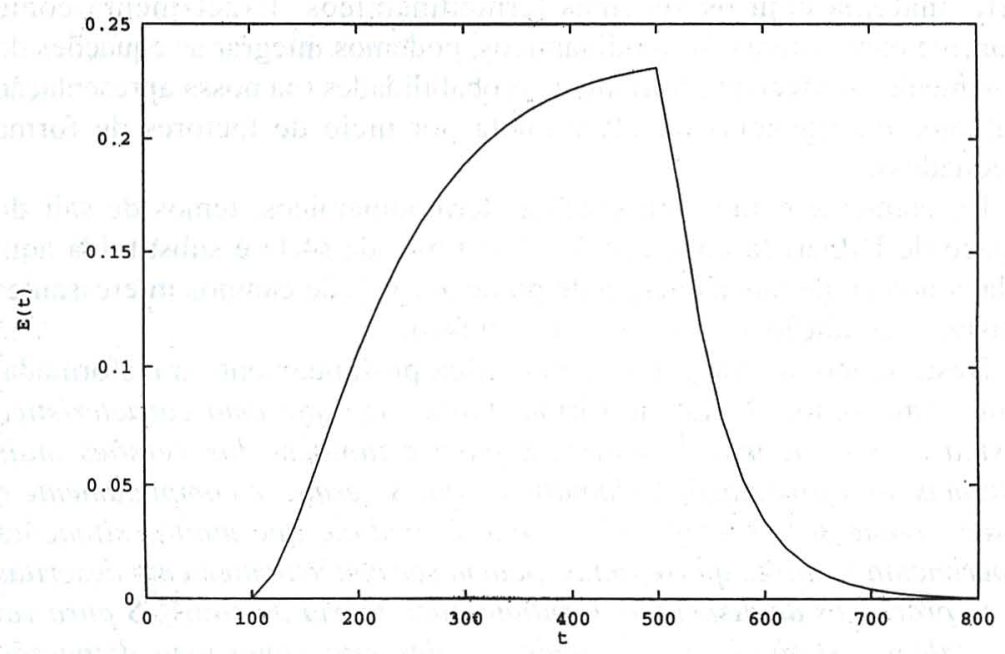

Fig. 8 - Formação e decaimento de partículas nuas enviando pacotes de ondas de forma rectangular no caso unidimensional

Este exemplo mostra que a nossa teoria é uma extensão da teoria quântica que evita a dificuldade enunciada por Dirac. É também interessante notar que a irreversibilidade aparece ao nível microscópico. As ressonâncias e os estados excitados estão claramente associados a estados instáveis. Encerremos esta secção com algumas notas sobre a relação entre campos e partículas.

Um problema fundamental da física moderna é a relação entre partículas e campos. Este é o problema fundamental da segunda quantificação. Uma teoria quântica livre é uma sobreposição de osciladores; para o campo electromagnético, as partículas correspondentes são os fotões. Temos $H=\sum_{\kappa} \omega_{\kappa}\left(n_{\kappa}+\frac{1}{2}\right)$. Campos quânticos livres são sistemas integráveis e os números de ocupação não são invariantes. Contudo, como não existem campos livres na natureza, as interacções 
conduzem a sistemas não integráveis onde os números de ocupação variam com o tempo.

Os campos não integráveis são sistemas com um número infinito de graus de liberdade com interacções persistentes. Existe, portanto, uma forte analogia com os sistemas termodinâmicos. Exactamente como acontece com sistemas termodinâmicos, podemos integrar as equações do movimento ao nível de Liouville de probabilidades (na nossa apresentação evitamos divergências no ultravioleta por meio de factores de forma adequados).

Exactamente como para sistemas termodinâmicos, temos de sair do espaço de Hilbert (a condição $N / V=$ finito de (4.1) é substituída aqui pela condição de que a energia de ponto zero $E_{0}$ de campos interessantes satisfaz a condição $V \rightarrow \infty, E_{0} / V=$ finito).

Deste modo, a teoria dos campos fica profundamente transformada. Num comentário, G. Kallen afirma: Parece-me que uma característica básica de uma teoria de matriz $\boldsymbol{S}$ pura e também das versões mais extremas da aproximação axiomática é que se esquece completamente o desenvolvimento no tempo. Claro que é verdade que muitas situações experimentais, talvez quase todas, podem ser convenientemente descritas como processos de dispersão. Contudo, uma teoria de matriz $\boldsymbol{S}$ pura vai mais além e supõe que tudo pode ser descrito como uma dispersão durante um intervalo de tempo infinito ${ }^{35}$. Podemos agora ultrapassar esta dificuldade e obter a equação da evolução válida para tempos finitos. Mais ainda: tal introduz a quebra da simetria temporal, o que explica o facto de a maior parte das partículas elementares ser instável.

\section{CONCLUSÃo}

O resultado principal do nosso trabalho é a formulação das leis microscópicas da natureza, que são expressas com base em probabilidades (e não trajectórias ou funções de onda) e conduzem a quebra de simetria temporal. Isto unifica a dinâmica clássica e a termodinâmica. Dá também uma nova formulação unificada à teoria quântica, uma vez que não necessitamos mais da formulação comum dual baseada na equação de Schroedinger suplementada pelo colapso da função de onda.

O sentido do tempo é uma característica comum a todos os objectos que pertencem ao nosso universo. Todos ficamos mais velhos no mesmo sentido, todas as estrelas e galáxias ficam mais velhas no mesmo sentido. Portanto, era um paradoxo estranho que o sentido do tempo não estivesse incorporado nas leis básicas da natureza. Contudo, as ferramentas para 
isso não estavam disponíveis no tempo de Boltzmann. Graças ao avanços da análise funcional e à teoria dos sistemas dinâmicos vemos agora que podemos formular as leis da natureza que capturam a irreversibilidade e dão à probabilidade um novo estatuto. A probabilidade já não é a expressão de um estado de espírito, mas reflecte uma propriedade da natureza que torna possível a emergência da vida. Não é uma falha da razão, mas o começo de uma nova racionalidade, na qual a razão não é identificada com a certeza nem a probabilidade com a ignorância.

\section{Agradecimentos}

O conteúdo deste artigo é o resultado de décadas de trabalho do grupo de Austin - Bruxelas. É impossível agradecer individualmente a cada membro do nosso grupo. Queremos, no entanto, fazer uma excepção para o Dr. I. Antoniou e para o Dr. G. Ordonez pelas suas contribuições. Ambos nos ajudaram a estruturar este artigo. Agradecemos ao Engineering Research Program, do Office of Basic Energy Sciences do Departamento de Energia dos EUA, bolsa n. ${ }^{\circ}$ DE-FG03-94ER14465, a bolsa F-0364 da Fundação Robert A. Welch, à Comissão Europeia, ao projecto CTIAC do Esprit, à DG III, à Lotaria Nacional da Bélgica e à Comunidade Francesa da Bélgica pelo apoio a este trabalho.

\section{Referências}

${ }^{1}$ Prigogine, I., From Being to Becoming. (Freeman, Nova Iorque, 1980). $/$ Gelfand, I. M.; Vilenkin, Ya N., Generalized Functions, vol. 4, (Academic Press, Nova Iorque, 1964); K. Maurin, Generalized Eigenfunctions, Expansions and Unitary Representations of Topological Groups. (Polish Scientific Publishers, Varsóvia, 1968); Gelfand, I.; Shilov, G., Generalized Eigenfunctions, vol. 3, Theory of Differential Equations. (Academic Press, Nova Iorque, 1967); Bohm, A., The Rigged Hilbert Space and Quantum Mechanics, Lecture Notes on Physics, vol. 78 (Springer, Berlim, 1986); Bohm, A.; Gadella, M.; Dirac K., Gamov vectors and Gelfand triplets. (Springer, Berlim, 1989); Bourbaki, N., Topological Vector Spaces. (Springer, Berlim, 1987).

${ }^{2}$ Gelfand, I. M.; Vilenkin, Ya N., Generalized Functions, vol. 4, (Academic Press, Nova Iorque, 1964); K. Maurin, Generalized Eigenfunctions, Expansions and Unitary Representations of Topological Groups. (Polish Scientific Publishers, Varsóvia, 1968); Gelfand, I.; Shilov, G., Generalized Eigenfunctions, vol. 3, Theory of Differential Equations. (Academic Press, Nova Iorque, 1967); Bohm, 
A., The Rigged Hilbert Space and Quantum Mechanics, Lecture Notes on Physics, vol. 78 (Springer, Berlim, 1986); Bohm, A.; Gadella, M.; Dirac K., Gamov vectors and Gelfand triplets. (Springer, Berlim, 1989); Bourbaki, N., Topological Vector Spaces. (Springer, Berlim, 1987).

${ }^{3}$ Citado em Birkhoff, G., Proceedings of Symposium in Price Mathematics, American Mathematical Society, vol. 2, p. 158.

${ }^{4}$ Prigogine, I., From Being to Becoming. (Freeman, Nova Iorque, 1980).

5 Idem, Ibidem.

${ }^{6}$ Koopman, B. O., Procedings of the National Academy of Sciences, USA 17, 315 (1931).

${ }^{7}$ Goodrich, K.; Gustafson, K.; Misra, B., Physica A 102, 379 (1980).

${ }^{8}$ Davies, E. B., Quantum Theory of Open Systems. (Academic Press, Landau, 1976).

${ }^{9}$ Arnold V.; Avez, A., Ergodic Problems in Classical Mechanics (Benjamin, Nova Iorque, 1968).

10 Idem.

11 Misra, B.; Prigogine, I.; Courbage, M., Proceedings of the National Academy of Sciences, USA 76, 3607 (1970).

12 Idem, Ibidem.

13 Suchanecki, Z.; Weron, A.; Rybaczak, M.; Weron K., Physica A 155, 385 (1984). / Antoniou, I.; Gustafson, K., Physica A 236, 296 (1987); Suchanecki, Z., Physica A 252, 345 (1998).

14 Prigogine, I., From Being to Becoming. (Freeman, Nova Iorque, 1980).

15 von Neumann, J.; Koopman, B., Proceedings of the National Academy of Sciences, USA 18, 255 (1932).

16 Antoniou, I.; Tasaki, S., International Journal of Quantum Chemistry A6, 425 (1993). / Driebe, Dean Fully Chaotic Maps and Broken Time Symmetry. (Kluwer Academic Publishers, 1999). Referências aos artigos originais são fornecidas neste trabalho.

17 Arnold V.; Avez, A., Ergodic Problems in Classical Mechanics (Benjamin, Nova Iorque, 1968).

18 Antoniou, I.; Tasaki, S., International Journal of Quantum Chemistry A6, 425 (1993).

19 Antoniou, I.; Tasaki, S., International Journal of Quantum Chemistry A6, 425 (1993). / Driebe, Dean Fully Chaotic Maps and Broken Time Symmetry. (Kluwer Academic Publishers, 1999). Referências aos artigos originais são fornecidas neste trabalho.

${ }^{20}$ Dirac, P. A., Lectures on Quantum Field Theory. (Belfer Grasd. School, Yeshiva University, Nova Iorque, 1996).

21 Petrosky, T.; Prigogine, I., Chaos, Solitons and Fractals, 7, 441 (1996). I Petrosky, T.; Prigogine, I., Extension of Classical Dynamics. The Case of Anharmonic Lattices, Gravity, Particles and Space-time, eds. P. Pronin; G. Sardanasshvily. (World Scientific, Singapore, 1996).

22 Prigogine, I., Non Equilibrium Statistical Mechanics (Wiley \& Sons, Nova Iorque, 1962).

${ }^{23}$ Idem.

${ }^{24}$ Idem.

25 Idem. 
26 Idem.

27 Petrosky, T.; Prigogine, I., Chaos, Solitons and Fractals, 7, 441 (1996). I

Petrosky, T.; Prigogine, I., Extension of Classical Dynamics. The Case of Anharmonic Lattices, Gravity, Particles and Space-time, eds. P. Pronin; G. Sardanasshvily. (World Scientific, Singapore, 1996).

28 Petrosky, T.; Prigogine, I., Chaos, Solitons and Fractals, 7, 441 (1996). I

Petrosky, T.; Prigogine, I., Advances in Chemical Physics, 99, 1 (1997).

29 Petrosky, T.; Prigogine, I., Chaos, Solitons and Fractals, 7, 441 (1996). I

Petrosky, T.; Prigogine, I., Advances in Chemical Physics, 99, 1 (1997).

30 Petrosky, T.; Prigogine, I., Advances in Chemical Physics, 99, 1 (1997). I

Petrosky, T.; Ordonez, G.; Miyasaka, T., Physical Review A 53, 4075 (1996). I

Petrosky, T.; Ordonez G., Physical Review A 563507 (1997).

31 Dirac, P. A., Principles of Quantum Mechanics (Oxford University Press,

4. ${ }^{\mathrm{a}}$ edição, 1962).

32 Petrosky, T.; Prigogine, I.; Tasaki, S., Physica A 173, 175 (1991).

33 Idem.

34 Dirac, P. A., Principles of Quantum Mechanics (Oxford University Press, 4. ${ }^{\text {a }}$ edição, 1962).

35 Källen, G., 12th Solvay Meeting, Outubro de 1961, p. 253. (Interscience, Nova Iorque). 
 \\ A palavra "fronteiras" pode ser tomada em} diferentes sentidos. Pode referir-se aos limites, necessariamente provisórios, entre o conhecido e o desconhecido, ou aos limites entre o possivel e o impossivel, e, dentro do possivel, entre o desejável e 0 indesejável. Fronteiras podem também ser as delimitações, nem sempre nítidas, entre ciência e não-ciência, e dentro da ciência, entre as várias disciplinas. Quais são então as fronteiras da ciência?

Neste livro, a resposta a esta pergunta é dada, segundo as mais diferentes perspectivas, por um conjunto notável de personalidades, cientistas ou não, entre as quais se contam três Prémios Nobel.

Rui Fausto, Carlos fiolhais e JoÃo Fillipe Queiró são, respectivamente, professores de Química, Física e Matemática na Faculdade de Ciências e Tecnologia da Universidade de Coimbra. 